\title{
The existence of eigenvalue problems for the waveguide theory
}

\section{A Maher $^{1 *}$ and EM Karachevskii ${ }^{2}$}

\section{"Correspondence:}

a_maher1969@yahoo.com

'Department of Mathematics,

Faculty of Science, Assiut University,

Assiut, 71516, Egypt

Full list of author information is

available at the end of the article

\begin{abstract}
In this paper, the existence of the eigenvalue problem for the waveguide theory is investigated. We used the Fourier transformation method for the solution of this problem. Also, we applied this problem to a dielectric waveguide. In this study, four theorems and two lemmas are obtained.
\end{abstract}

MSC: $35 \mathrm{~A} 22 ; 35 \mathrm{P} 10$

Keywords: partial differential equations; eigenvalue problems; Fourier transformation method

\section{Basic preliminaries}

A dielectric waveguide is a composite of its own index of refraction for each layer. If $\Omega_{j}$ is a layer, where the index of refraction is $k_{j}$ and $\mu$ is a spectral parameter, then the waveguide process can be written in the following form:

$$
-\triangle u_{j}(x)=\left(k_{j}+\mu\right) u_{j}(x) ; \quad x \in \Omega_{j}
$$

where

$$
\Delta=\left(\frac{\partial}{\partial x_{1}}\right)^{2}+\cdots+\left(\frac{\partial}{\partial x_{n}}\right)^{2} .
$$

In order to obtain $u_{j}(x)$ and $u_{r}(x)$, the process in all the waveguide for the common boundary of domains $\Omega_{j}$ and $\Omega_{r}$ is evaluated. $u_{j}(x)$ and $u_{r}(x)$ must be joined in the way that the obtained known functions $u_{j, r}(x)=u_{j}(x)$ for $x \in \Omega_{j}$ and $u_{j, r}(x)=u_{r}(x)$ for $x \in \Omega_{r}$ will be the generalized solution of the equation

$$
-\triangle v(x)=(g(x)+\mu) v(x) ; \quad x \in \Omega_{j} \cup \Omega_{r},
$$

in which $g(x)=k_{j}$ for all $x \in \Omega_{j}$ and $g(x)=k_{r}$ for all $x \in \Omega_{r}$. If the boundary $\Gamma_{j, r}$ is sufficiently smooth, the condition of this junction may be put down in a natural form. Indeed, the contraction of $\Gamma_{j, r}$ is noninfinitely smooth in $\Omega_{j}$ and $\Omega_{r}$, the functions which deteriorate their smoothness where the conditions themselves could be impossible to write. That is how the solution of this problem was progressing.

If the boundaries of domains are bad and there are several of them, it is not clear what the condition of the junction looks like. In this situation (connection), we need another approach to the solution of the set problem.

(C) 2012 Maher and Karachevskii; licensee Springer. This is an Open Access article distributed under the terms of the Creative Commons Attribution License (http://creativecommons.org/licenses/by/2.0), which permits unrestricted use, distribution, and reproduction in any medium, provided the original work is properly cited. 
Since results of the junction must preserve the property of solution (being a generalized solution), we propose a new circuit system to solve the set problem. In general case, it is not solved.

The existence of eigenvalue is proved in [1] for the special case $n=2, N=2, \Omega_{1}$ - the circle. For more details, see [2-5] and [6].

Consider the problem

$$
-\triangle u(x)=(g(x)+\mu) u(x) ; \quad x \in R^{n}, u \in L^{2}\left(R^{n}\right),
$$

in which $g(x)=k_{j}$ for all $x \in \Omega_{j}$, and

$$
R^{n}=\bigcup_{j=1}^{N}\left(\Omega_{j} \cup \Gamma_{j}\right) .
$$

It is obvious that if we prove the existence of the eigenvalue (3), we obtain the following solution of the problem (1) $u_{j}(x)=u(x) ; x \in \Omega_{j}, j=1, \ldots, N$, where they are found automatically joined by a required form.

\section{Formulation of the problem}

We consider the eigenvalue problem (3) where $R^{n}=\bigcup_{j=1}^{N}\left(\Omega_{j} \cup \Gamma_{j}\right)$ and $\Omega_{j}, j=1, \ldots, N$ are mutually exclusive (disjoint) measurable sets with a positive measure. If we introduce a new spectral parameter $\lambda=\mu+k_{j}$, then the problem (1) takes the form

$$
-\triangle u(x)=(c(x)+\lambda) u(x) ; \quad x \in R^{n}, u \in L^{2}\left(R^{n}\right),
$$

in which $c(x)=0$, if $x \in \Omega_{j}$.

The problem (5) is self-adjoint. This can be easily seen if we use the Fourier transformation. However, it does not influence the eigenvalue existence. Some examples of the problem (5) are known (with concrete $k_{j}, N$ and $\Omega_{j}$ ) both with and without eigenvalues.

To use the Fourier transformation $(F u(x))(z)$ of the distribution (generalized) function $u(x)$ of slow growth, we must be aware of the following well-known Parseval equality:

$$
\int(F u(x))(z)(F v(x))(z) d z=\int u(x) v(z-x) d x,
$$

and Plancherel's theorem: $(F v(x))(z) \in L^{2}\left(R^{n}\right)$ if and only if $v \in L^{2}\left(R^{n}\right)$, where

$$
\|v(x)\|=\|(F v(x))(z)\|,
$$

for all $u$ and $v \in L^{2}\left(R^{n}\right)$.

From now on, if it is not specifically indicated, the notation $\|\cdot\|$ is the norm in the space $L^{2}\left(R^{n}\right)$.

\section{The existence of negative eigenvalues for the general case}

Let us consider the problem:

$$
P(D) u(x)=(c(x)+\lambda) u ; \quad x \in R^{n}, u(x) \in L^{2}\left(R^{n}\right),
$$


in which $c(x)$ is a measurable function, $c(x)>0$ for all $x \in \Omega,|c(x)| \leq d<+\infty$ almost everywhere in $\Omega, c(x)=0$ outside $\Omega, \Omega$ is measurable and $P(D)$ is a linear pseudo-differential operator with constant coefficients. Here $P(i z) \geq$ argument quasi-polynomial $z \in R^{n}$, not depending on $x$ and satisfying the following conditions for all $z \in R^{n}$ :

$$
\begin{aligned}
& P(i z) \geq 0, \quad P(i z)=P(-i z) \quad \text { for all } z, \\
& {[P(i z)-\mu]^{-1} \in L^{2}\left(R^{n}\right) ; \quad \mu<0,} \\
& \int_{R^{n}}|P(i z)-\mu|^{-2} d z \rightarrow 0, \quad \text { if } \mu \rightarrow-\infty .
\end{aligned}
$$

We suppose that

$$
\int_{|z| \leq \delta}|P(i z)-\mu|^{-2} d z \rightarrow+\infty, \quad \text { if } \mu \rightarrow 0^{-}
$$

for each sufficiently small $\delta>0$ and $\mu \rightarrow 0^{-}$.

Theorem 1 The problem (6) has at least one negative eigenvalue if $\Omega$ is bounded.

It is necessary to introduce several lemmas before proving this theorem.

In each case, we consider $\mu<0$. By virtue of (8), there is a function $h(x, \mu) \in L^{2}\left(R^{n}\right)$ of the Fourier transformation which coincides with $[P(i z)-\mu]^{-1}$. Considering $(7)$, the real and even function $h(x, \mu)$ could be obtained.

Lemma 1 Let $\mu<0$. The problem (6) has a nonzero solution if and only if the nonzero solution $v(x)$ has the form

$$
v(x)=\int_{\Omega} c(t) v(t) h(x-t, \mu) d t, \quad v \in L^{2}(\mu) .
$$

Proof Applying the Fourier transformation for (6) yields

$$
(P(i z)-\mu)(F u(x))(z)=[F(c(x) u(x))](z) \in L^{2}\left(R^{n}\right) .
$$

Hence, in particular, the integral

$$
\int(F(u(x)))(z) e^{i x z} d z
$$

converges absolutely. From now on, $i x z=\left(i x_{1} z_{1}, \ldots, i x_{n} z_{n}\right)$. It follows from latter relations

$$
u(x)=\frac{1}{\sqrt{2 \pi}} \int[F(u(x))](z) e^{i x z} d z=\int[F(c(t) u(t))](z)\left[F_{t} h(x+t, \mu)\right](z) d z,
$$

where $F_{t}$ means that the Fourier transformation has been determined under $t$. Hence, by virtue of Parseval's equality, it follows that

$$
u(x)=\int c(t) u(t) h(x-t, \mu) d t .
$$


Since $c(t)=0$ outside $\Omega$, then $u(x) ; x \in \Omega$ is the solution of the problem (11). If $u(t)=0$ where in $\Omega$ we obtain $[c(t) u(t)]=0$ for $R^{n}$, by virtue of the latter equality $u(x)=0$. The necessity is proved.

Let us prove the sufficiency. Let $v(x)$ be the nonzero solution of the problem (11). Consider the new problem

$$
P(D) u=\mu u+c(x) f(x), \quad x \in R^{n} ; u \in L^{2}\left(R^{n}\right),
$$

in which $f(x)=v(x)$ for all $x \in \Omega$ and $f(x)=0$ outside $\Omega$. Since $c(x) f(x) \in L^{2}\left(R^{n}\right)$, applying the Fourier transformation for (12), we obtain

$$
\begin{aligned}
& {[P(i z-\mu)][F u(x)](z)=[F(c(x) f(x))] z \in L^{2}\left(R^{n}\right) ;} \\
& u(x)=\frac{1}{\sqrt{2 \pi}} \int[F(c(x) f(x))](z)\left[F_{t} h(x+t, \mu)\right](z) d z=\int c(t) f(t) h(x-t, \mu) d t .
\end{aligned}
$$

From Parseval's equality, the solution of the problem (12) exists and it is unique. In particular, when $x \in \Omega$, we have

$$
u(x)=\int_{\Omega} c(t) v(t) h(x-t, \mu) d t=v(x) .
$$

Considering this inequality and (12), we obtain $c(x) f(x)=c(x) u(x)$, i.e., $u(x)$ is the solution of the problem (6). Thus, the lemma is proved.

In the case when $\mu<0$, we consider $A(\mu)$ as an integral operator, where

$$
A(\mu) \omega(x)=\sqrt{c(x)} \int_{\Omega} \sqrt{c(t)} h(x-t, \mu) \omega(t) d t ; \quad \omega \in L_{b}^{2}(\Omega) .
$$

We remember that the operator $A(\mu)$ is defined only when $\mu<0$. Since $P(i z)=P(-i z)$, thus the Fourier transformation for the functions $h(t, \mu), h(-t, \mu)$ coincides. That is why $h(t, \mu)=h(-t, \mu)$. If $\Omega$ is bounded, then the kernel $(\sqrt{c(x)} \sqrt{c(t)} h(x-t, \mu))$ of the integrated operator $A(\mu)$ belongs to $L_{b}^{2}(\Omega \times \Omega)$. It follows that the operator $A(\mu)$ is completely continuous. Its self-adjointness and positiveness are obvious. This enables us to write down the eigenvalues of the operator $A(\mu)$ :

$$
\lambda_{1}(\mu)>\lambda_{2}(\mu)>\cdots
$$

It is well known that (see [7])

$$
\lambda_{1}(\mu)=\operatorname{Sup}<A(\mu) f
$$

where Sup is determined for all the function $f \in L_{b}^{2}(\Omega)$, for which $\|f\| \leq 1$.

From the known results for self-adjoint and quite continuous operators (see [7]), it follows that $\lambda_{k}(\mu)$ continuously depends on $\mu$, where

$$
\left|\lambda_{1}(\mu)\right|^{2}=\|A(\mu)\|^{2}=\int_{\Omega} \int_{\Omega} c(x) c(t)(h(x-t, \mu))^{2} d x d t .
$$


Lemma 2 Let $\Omega$ be bounded when $x \in \Omega$. Then

(1) $\lambda_{k}(\mu) \rightarrow 0$ at $\mu \rightarrow-\infty$,

(2) $\lambda_{1}(\mu) \rightarrow+\infty$ at $\mu \rightarrow 0^{-}$.

Proof Since $\lambda_{j}(\mu) f_{j}(x, \mu)=A(\mu) f(x, \mu),\left\|f_{j}(x, \mu)\right\|_{\Omega}=1$, and $|c(x)| \leq a<+\infty$, we have

$$
\begin{aligned}
\left|\lambda_{j}(\mu)\right| & \leq\|A(\mu)\|=\left[\int_{\Omega} \int_{\Omega} c(x) c(t)(h(x-t, \mu))^{2} d x d t\right]^{\frac{1}{2}} \\
& \leq a\left[\int_{\Omega} c(t)\left(h(x-t, \mu)^{2} d x\right) d t\right]^{\frac{1}{2}} \\
& =a\|h(x, \mu)\| \cdot\left(\int_{\Omega} c(t) d t\right)^{\frac{1}{2}} \\
& \leq a\|h(x, \mu)\| \cdot\|c(t)\|_{\Omega} \\
& =a_{1}\left(\int \frac{d z}{(P(i z)-\mu)^{2}}\right)^{\frac{1}{2}} .
\end{aligned}
$$

Hence, the first statement follows from (9).

Let us prove the second statement. By virtue of (13), with $c(x)=0$ outside $\Omega$ and

$$
\left(\lambda_{1}(\mu)\right)^{2}=\iint[c(x) h(x-t, \mu)] \cdot[c(t) h(x-t, \mu)] d x d t,
$$

which is applied to the last integral in Parseval's inequality, we obtain

$$
\left(\lambda_{1}(\mu)\right)^{2}=\iint(F[c(x) h(x-t, \mu)])(z, \xi) \cdot(F[c(t) h(x-t, \mu)])(-z,-\xi) d z d \xi .
$$

The following equations are correct:

$$
\begin{aligned}
(F[c(x) h(x-t, \mu)])(z, \xi) & =\left(F_{x}\left[c(x) \cdot \frac{e^{-i x \xi}}{P(i \xi)-\mu}\right]\right)(z, \xi) \\
& =\frac{1}{P(i \xi)-\mu}\left(F_{x}\left[c(x) e^{-i x \xi}\right]\right)(z, \xi) \\
& =\frac{1}{\sqrt{2 \pi}} \cdot \frac{1}{P(i \xi)-\mu} \int_{\Omega} c(x) e^{-i x(\xi+z)} d x .
\end{aligned}
$$

In a similar way, we obtain

$$
(F[c(t) h(x-t, \mu)])(-z,-\xi)=\frac{1}{\sqrt{2 \pi}} \cdot \frac{1}{P(-i \xi)-\mu} \int_{\Omega} c(t) e^{i t(\xi+z)} d t .
$$

Thus, we have proved the following:

$$
\left(\lambda_{1}(\mu)\right)^{2}=\frac{1}{2 \pi} \iint\left|\int_{\Omega} c(x) e^{-i x(\xi+z)} d x\right|^{2} \cdot \frac{d z d \xi}{[P(i \xi-\mu)]^{2}} .
$$

The following estimate is obvious:

$$
\left(\lambda_{1}(\mu)\right)^{2} \geq \frac{1}{2 \pi} \int_{|z| \leq \delta} \int_{|\xi| \leq \delta}\left|\int_{\Omega} c(x) e^{-i x(\xi+z)} d x\right|^{2} \cdot \frac{d z d \xi}{[P(i \xi)-\mu]^{2}},
$$


where

$$
\left|\int_{\Omega} c(x) e^{-i x(\xi+z)} d x\right|^{2}=\left|\int_{\Omega} c(x) d x+\int_{\Omega} c(x)\left[e^{-i x(\xi+z)}\right] d x\right|^{2}
$$

$\delta$ will be chosen in a way such that $\left|e^{-i x(z+\xi)}-1\right| \leq \frac{1}{2}$ for all $x \in \Omega$ and $|z| \leq \delta,|\xi| \leq \delta$. Since $\Omega$ is bounded, we may always obtain the latter.

Considering (16) and (17), we obtain

$$
\begin{aligned}
\left(\lambda_{1}(\mu)\right)^{2} & \geq \frac{1}{4 \cdot 2 \pi}\left(\int_{\Omega} c(x) d x\right)^{2} \cdot \int_{|z| \leq \delta} d z \int_{|\xi| \leq \delta} \frac{d \xi}{[P(i \xi-\mu)]^{2}} \\
& =b_{1}(\delta) \int_{|z| \leq \delta} \frac{d \xi}{(P(i \xi)-\mu)^{2}} .
\end{aligned}
$$

Hence, by virtue of (10), the lemma is proved.

Proof of Theorem 1 At the first stage, we suppose that $c(x) \geq v>0$ for all $x \in \Omega$. By virtue of Lemmas 1 and 2, where $\lambda_{1}\left(\mu_{0}(v)\right)=1$ for $\mu_{0}(v)<0$, if $f_{1}(x)$ is the eigenfunction corresponding to the eigenvalue $\lambda_{1}\left(\mu_{0}(v)\right)$, then

$$
\phi(x)=f_{1}(x) / \sqrt{c(x)} \in L^{2}(\Omega) .
$$

When $\mu=\mu_{0}(v)$, we have the nonzero solution of the equation (11). It follows from Lemma 1 that $\mu_{0}(v)$ is the eigenvalue of the problem (6).

For the general case, we put $c(v, x)=c(x)$ if $x \in \Omega$ and $c(x) \geq v ; c(v, x)=v$ when $x \in \Omega$ and $c(x) \in(0, v)$. The nonzero solutions of the equation

$$
\phi(v, x)=\int_{\Omega} c(v, x) h\left(x-t, \mu_{0}(v)\right) \phi(v, t) d t
$$

are chosen in such a way that $\|\phi(\nu, x)\|_{\Omega}$.

The integral operators defined by the right-hand sides of (11) and (18) are defined in $B(\mu), B\left(v, \mu_{0}(v)\right)$ respectively. Since $\Omega$ is bounded, then both $\{c(v, x)\}$ and $\left\{h\left(x, \mu_{0}(v)\right)\right\}$ uniformly converge by norm to $c(x)$ and $h\left(x, \mu_{0}\right)$ respectively. If $\mu_{0}(v) \rightarrow \mu_{0}$, then

$$
\left\|B\left(\mu_{0}\right)-B\left(v, \mu_{0}(v)\right)\right\| \rightarrow 0 \quad \text { when } \mu_{0}(v) \rightarrow \mu_{0} .
$$

Considering the choice $\phi(v, x)$ and the property $\|h(x, \mu)\| \rightarrow 0$, if $\mu \rightarrow-\infty$, we can easily prove the boundedness of $\mu_{0}(v)$. Noting that when $\mu_{0}$ and $v_{0} \rightarrow 0$ for which $\mu_{0}\left(v_{j}\right) \rightarrow \mu_{0}$, the operator $B\left(\mu_{0}\right)$ is completely continuous. In this case, as we know, the set $B\left(\mu_{0}\right), \phi(\nu, x)$ contains the subsequence $B\left(\mu_{0}\right), \phi\left(v_{j}, x\right)$ which converges by norm where $\mu \rightarrow \mu_{0}$.

From (18) and (19) it follows that $\left\{\phi\left(v_{j}, x\right)\right\}$ converges to $\phi(x)$ by norm where $\|u(x)\|_{\Omega}=1$. Then $\left\{B\left(\nu, \mu_{0}\left(v_{j_{1}}\right)\right) \phi\left(v_{j_{1}}, x\right)\right\}$ converges to $B\left(\mu_{0}\right) \phi(x)$ by norm and satisfies the equality $u(x)=B\left(\mu_{0}\right) \phi(x)$, i.e., when $\mu=\mu_{0}$, the equation (11) has a nonzero solution. Hence, the theorem is proved. 


\section{Application to the problem of a dielectric waveguide}

In the case of

$$
\begin{aligned}
P(i z) & =-\left(i z_{1}\right)^{2}-\left(i z_{2}\right)^{2}-\cdots-\left(i z_{n}\right)^{2} \\
& =\left|z_{1}\right|^{2}+\left|z_{2}\right|^{2}+\cdots+\left|z_{n}\right|^{2}=|z|^{2} \geq 0,
\end{aligned}
$$

where

$$
P(i z)=P(-i z) \quad \text { for all } z \in R^{n},
$$

the condition (7) takes the form

$$
\int_{R^{n}}\left(|z|^{2}-\mu\right)^{-2} d z<+\infty \quad \text { when } \mu<0 .
$$

It is clear that in the case of $n$ arbitrary, these requirements are not satisfied. However, it takes place in the case $n \leq 3$ important for the application. It can easily be proved when we use the spherical coordinates. Moreover, for the case when $n \leq 3$, (9) also takes place. Let us make sure that (10) is satisfied when $n \leq 4$.

Let

$$
I=\int_{|z| \leq \delta} \frac{d z}{\left(|z|^{2}-\mu^{2}\right)^{2}} .
$$

Consider the spherical coordinates

$$
\begin{aligned}
& z_{1}=r \cos \theta_{1}, \quad z_{2}=r \sin \theta_{1} \cos \theta_{2}, \\
& z_{n-1}=r \sin \theta_{1} \cdots \sin \theta_{n-2} \cos \theta_{n-1}, \\
& z_{n}=r \sin \theta_{1} \sin \theta_{2} \cdots \sin \theta_{n-2} \sin \theta_{n-1} .
\end{aligned}
$$

The left-hand side of (20) takes the form

$$
\begin{aligned}
& \int_{0}^{+\infty} r^{n-1} \int_{c(\theta)}\left(r^{2}-\mu\right)^{-2}\left(\sin \theta_{1}\right)^{n-2}\left(\sin \theta_{2}\right)^{n-3} \cdots\left(\sin \theta_{n-2}\right) \cdot d r d \theta_{1} d \theta_{2} \cdots d \theta_{n-1} \\
& \quad=a_{2} \int_{0}^{+\infty} r^{n-1}\left(r^{2}-\mu\right)^{-2} d r,
\end{aligned}
$$

where

$$
c(\theta)=\left\{\theta_{1} \in[0,2 \pi], \theta_{j} \in\left[\frac{-\pi}{2}, \frac{\pi}{2}\right], j=2, \ldots, n-1\right\} .
$$

It follows that

$$
I=\int_{0}^{\delta} \int_{c(\theta)} \frac{r^{n-1} \sin ^{n-2} \theta_{1} \sin ^{n-3} \theta_{2} \cdots \sin \theta_{n-2}}{\left(r^{2}-\mu\right)^{2}} d r d \theta_{1} \cdots d \theta_{n},
$$

where

$$
c(\theta)=\left\{0 \leq \theta_{1} \leq 2 \pi, \frac{-\pi}{2} \leq \theta_{j}<\frac{\pi}{2}, j=2, \ldots, n\right\} .
$$


Hence,

$$
I=b_{2} \int_{0}^{\delta} \frac{r^{n-1}}{\left(r^{2}-\mu\right)^{2}} d r
$$

We can see that when $n \leq 4$,

$$
\int_{0}^{\delta} \frac{r^{n-1}}{\left(r^{2}-\mu\right)^{2}} d r \rightarrow+\infty ; \quad \mu \rightarrow-0
$$

Taking into account that (10) is satisfied and denoting index $j_{m}$ in which $k_{j}$ is the minimum, the problem (5) can be rewritten in the following form:

$$
-\Delta u(x)=\left[\left(g(x)-k_{j_{m}}\right)+\left(\mu+k_{j_{m}}\right)\right] u(x), \quad x \in R^{n}, u(x) \in L^{2}\left(R^{n}\right)
$$

when $g(x)-k_{j_{m}}=c(x)=0$ for all $x \in \Omega_{j_{m}} ;$ i.e., $c(x)=0$, outside $\left(R^{n}-\Omega_{j_{m}}\right)=\Omega$, where $c(x)>0$ at $x \in \Omega$.

The theorem may be applied to the problem (21). As a consequence of this theorem, we get the following:

Theorem 2 If $\Omega$ is bounded, the problem (3) has an eigenvalue $\mu$ for which $\mu+k_{j_{m}}<0$.

Let $j_{M}$ be the index at which $k_{j}$ is maximum. Then the problem (3) may take the form

$$
-\triangle u(x)=\left[\left(g(x)-k_{j_{M}}\right)+\left(\mu+k_{j_{M}}\right)\right] u(x) ; \quad x \in R^{n}, u(x) \in L^{2}\left(R^{n}\right)
$$

when

$$
g(x)-k_{j_{k}}<0, \quad \text { if } x \in \Omega_{j_{M}}
$$

and

$$
g(x)-k_{j_{k}}=0, \quad \text { if } x \in \Omega_{j_{M}} .
$$

Now, we formulate the following theorem.

Theorem 3 The problem (3) does not have an eigenvalue $\mu$ for which $\mu+k_{j_{M}} \leq 0$.

Proof Multiplying the equality (22) by $u(x)$ and integrating it in $R^{n}$, we have

$$
\begin{aligned}
\int(-\Delta u(x)) \cdot u(x) d x & =\int\left[\left(\frac{\partial}{\partial x_{1}}\right)^{2}+\cdots+\left(\frac{\partial}{\partial x_{n}}\right)^{2}\right] u(x) d x \\
& =\int\left(g(x)-k_{j_{M}}\right)(u(x))^{2} d x+\int\left(\mu+k_{j_{M}}\right)(u(x))^{2} d x .
\end{aligned}
$$

If $g(x)-k_{j_{M}} \leq 0, \mu+k_{j_{M}} \leq 0$, then by virtue of the condition $u(x)=0$, the latter is not impossible.

By virtue of Theorems 2 and 3, we have 
Theorem 4 Let $\left(R^{n}-\Omega_{j_{m}}\right)$ be bounded. Then the problem (3) has an eigenvalue $\mu$ which satisfies the condition $-k_{j_{M}} \leq \mu<-k_{j_{m}}$.

Remark If the condition that the bounded set $\left(R^{n}-\Omega_{j_{m}}\right)$ is not valid, then the problem may not have eigenvalues.

\section{Conclusions}

This paper deals with the existence of eigenvalue problems for the waveguide theory. These problems are very important in the study of the mathematical analysis and mathematical physics. In this paper, we introduced four theorems and two lemmas.

Competing interests

The authors declare that they have no competing interests.

Authors' contributions

The idea of this paper was introduced by the first author. The second author shared the first author in calculations.

\section{Author details}

${ }^{1}$ Department of Mathematics, Faculty of Science, Assiut University, Assiut, 71516, Egypt. ${ }^{2}$ Department of Applied

Mathematics, Kazan State University, 18 Kremlyovskaya st., Kazan, 420008, Russia.

\section{Acknowledgements}

We wish to thank the referees for their valuable comments which improved the original manuscript.

Received: 5 April 2012 Accepted: 1 November 2012 Published: 13 November 2012

\section{References}

1. Karchevskii, EM: The research (investigation) of the numerical method of solving the spectral problem for the theory of dielectric waveguides. Izv. Vysš. Učebn. Zaved., Mat. 1, 10-17 (1999)

2. Dautov, RZ, Karchevskii, EM: Existence and properties of solutions to the spectral problem of the dielectric waveguide theory. Comput. Math. Math. Phys. 40, 1200-1213 (2000)

3. Karchevskii, EM: The fundamental wave problem for cylindrical dielectric waveguides. Differ. Equ. 36, 998-999 (2000)

4. Karchevskii, EM, Solov'ev, SI: Investigation of a spectral problem for Helmholtz operator on the plane. Differ. Equ. 36, 563-565 (2000)

5. Pinasco, JP: Asymptotic of eigenvalues and lattice points. Acta Math. Sin. Engl. Ser. 22(6), 1645-1650 (2000)

6. Snyder, A, Love, D: Optical Waveguide Theory. Chapman \& Hall, New York (1987)

7. Riss, F, Sekefalvi-Nad, B: Lectures on Functional Analysis. Mir, Moscow (1979)

\section{Submit your manuscript to a SpringerOpen ${ }^{\ominus}$ journal and benefit from:}

- Convenient online submission

- Rigorous peer review

- Immediate publication on acceptance

- Open access: articles freely available online

- High visibility within the field

- Retaining the copyright to your article 